\title{
Is Routine Preoperative Esophagogastroduodenoscopy Prior to Bariatric Surgery Mandatory? Systematic Review and Meta-analysis of 10,685 Patients
}

\author{
Walid El Ansari $^{1,2,3}$ (D) Ayman El-Menyar ${ }^{4,5} \cdot$ Brijesh Sathian $^{4} \cdot$ Hassan Al-Thani $^{6} \cdot$ Mohammed Al-Kuwari $^{7}$. \\ Abdulla Al-Ansari ${ }^{1}$
}

Published online: 28 May 2020

(C) The Author(s) 2020

\begin{abstract}
Background This systematic review and meta-analysis searched, retrieved and synthesized the evidence as to whether preoperative esophagogastroduodenoscopy (p-EGD) should be routine before bariatric surgery (BS).

Methods Databases searched for retrospective, prospective, and randomized (RCT) or quasi-RCT studies (01 January 2000-30 April 2019) of outcomes of routine p-EGD before BS. STROBE checklist assessed the quality of the studies. P-EGD findings were categorized: Group 0 (no abnormal findings); Group 1 (abnormal findings that do not necessitate changing the surgical approach or postponing surgery); Group 2 (abnormal findings that change the surgical approach or postpone surgery); and Group 3 (findings that signify absolute contraindications to surgery). We assessed data heterogeneity and publication bias. Random effect model was used.

Results Twenty-five eligible studies were included (10,685 patients). Studies were heterogeneous, and there was publication bias. Group 0 comprised 5424 patients (56\%, 95\% CI: 45-67\%); Group 1, 2064 patients (26\%, 95\% CI: 23-50\%); Group 2, 1351 patients (16\%, 95\% CI: $11-21 \%)$; and Group 3 included 31 patients $(0.4 \%, 95 \%$ CI: $0-1 \%)$.

Conclusion For $82 \%$ of patients, routine p-EGD did not change surgical plan/ postpone surgery. For 16\% of patients, p-EGD findings necessitated changing the surgical approach/ postponing surgery, but the proportion of postponements due to medical treatment of H Pylori as opposed to "necessary" substantial change in surgical approach is unclear. For 0.4\% patients, p-EGD findings signified absolute contraindication to surgery. These findings invite a revisit to whether p-EGD should be routine before $\mathrm{BS}$, and whether it is judicious to expose many obese patients to an invasive procedure that has potential risk and insufficient evidence of effectiveness. Further justification is required.
\end{abstract}

Keywords Preoperative $\cdot$ Esophagogastroduodenoscopy $\cdot$ Laparoscopic sleeve gastrectomy $\cdot$ Bariatric surgery

Walid El Ansari

welansari9@gmail.com

Ayman El-Menyar

aymanco65@yahoo.com

Brijesh Sathian

BSathian@hamad.qa

Hassan Al-Thani

althanih@hotmail.com

Mohammed Al-Kuwari

malkuwari2@hamad.qa

Abdulla Al-Ansari

AALANSARI1@hamad.qa
1 Department of Surgery, Hamad Medical Corporation, Doha, Qatar

2 College of Medicine, Qatar University, Doha, Qatar

3 Schools of Health and Education, University of Skovde, Skövde, Sweden

4 Department of Surgery, Trauma and Vascular Surgery, Clinical Research, Hamad General Hospital, Doha, Qatar

5 Clinical Medicine, Weill Cornell Medical School, Doha, Qatar

6 Department of Surgery, Trauma and Vascular Surgery Section, Hamad Medical Corporation, Doha, Qatar

7 Department of Bariatric Surgery, Hamad Medical Corporation, Doha, Qatar 


\section{Introduction}

There is a debate about the utility of routine preoperative esophagogastroduodenoscopy (p-EGD) screening of patients undergoing bariatric surgery (BS) $[1,2]$. The European and Italian national recommendations advocate the use of presurgery upper gastrointestinal endoscopy together with multiple biopsies in the work-up of patients; conversely, the American Society for Metabolic \& Bariatric Surgery only recommends it in selected cases with symptomatic gastric disease [3-5]. Generally, the question of routine p-EGD has many clinical implications and significant financial repercussions [1].

Some evidence supports routine p-EGD among patients undergoing BS. The reasons include the weak correlation between the patients' symptoms and p-EGD findings, that p-EGD is convenient, safe, applied easily [6-8], and pEGD findings may alter the management and hence eliminate the future development of gastric pathology [9], or detect asymptomatic benign or pre/malignant lesions. Missing asymptomatic lesions in some BS where the distal stomach and/or duodenum is rendered unreachable by esophagogastroduodenoscopy could lead to missing some lesions in the bypassed stomach that p-EGD could have discovered [10-16]. Some authors endorse that all BS patients have p-EGD, as after surgery, the endoscope may not reach the gastric/duodenal mucosa [17]. In agreement, others recommended that all BS patients should have upper gastrointestinal endoscopy [8]. For some procedures (e.g., laparoscopic adjustable gastric banding and vertical banded gastroplasty), $\mathrm{p}$-EGD could provide information that might influence the operative procedure, particularly due to upper gastrointestinal lesions that often require medical therapy $[7,18]$.

It remains contested whether routine p-EGD should be undertaken for all patients undergoing e.g., laparoscopic sleeve gastrectomy (LSG) [19]. Some authors support routine pEGD in patients with upper gastrointestinal symptoms (symptomatic cases only) [3, 20,21]. Others suggest a selective approach for asymptomatic cases, because of the weak clinical relevance of most lesions discovered on routine p-EGD, its cost, and invasiveness [22, 23]. Still, other research found that routine p-EGD in LSG might require further justification for asymptomatic patients due to its low utility in managing such patients in regions with low prevalence of upper gastrointestinal cancers [2]. Only $2 \%$ of asymptomatic patients had any abnormality detected at p-EGD, none of which affected their treatment plan, and hence a focus on symptomatic patients only can safely reduce $\mathrm{p}$-EGD rate by $80 \%$ [24].

Others reported that most of the pathology identified at $p$ EGD among patients scheduled for gastric banding did not significantly influence their management; however, two early cancers were detected [25]. In addition, although obesity is a risk factor for gastroesophageal reflux and esophageal adenocarcinoma, research could not confirm a high prevalence of Barrett's esophagus among 233 patients selected for laparoscopic gastric banding [26]. Likewise, the association between obesity and reflux remains controversial [27], and it is unclear whether BS impacts the advancement of gastroesophageal reflux disease (GERD) [28]. Despite a somewhat inaccessible foregut after bypass surgery, the low gastric cancer incidence among Caucasians [29] may not demand routine p-EGD [30].

Opinions remain divided as to whether p-EGD should be undertaken for all BS patients. One position is that the "intuitive reasons to continue p-EGD screening of BS patients include endoscopic findings that optimize medical management for the healing of their BS in a substantial proportion of patients and/or the endoscopic findings in at least a few patients that alter or delay the surgery itself" (p. 712) [22]. Conversely, others recommended that standard p-EGD is not indicated, as many BS patients are screened in order to discover clinically significant abnormalities [11]. For example, in Turkey, none of the 755 LSG patients had macro/microscopic malignant pathological finding in the preoperative upper gastrointestinal endoscopy [31]. In Brazil, researchers did not perform routine p-EGD on 649 LSG patients and only did when patients complained of abdominal pain or dysphagia; however, even with these symptomatic complaints, most patients had no abnormal findings [32]. Across $93.2 \%$ of BS patients, p-EGD findings were negative or had no effect on the preoperative management or choice of surgery; thus, it might not be wise to expose morbidly obese patients to a routine invasive uncomfortable procedure that carries potential (although minimal) risk [21]. Hence, authors have raised the question: "We do not screen the general population for those minor esophagogastroduodenoscopy findings; so why should we do it on people planned for bariatric surgery?" (p. 414) [21]. Likewise, a comment on "Is esophagogastroduodenoscopy before Roux-en-Y gastric bypass or sleeve gastrectomy mandatory?" concluded that $\mathrm{p}$ EGD had no value in prediction or prevention of postoperative complications [33].

Such inconsistency highlights a gap as to whether routine p-EGD is sufficiently justified for all BS patients, and inspired the current systematic review and meta-analysis of the significance of routine p-EGD screening in BS. To the best of our knowledge, there exists no systematic review of the English literature on the topic, and no meta-analysis has been undertaken to answer this important question. Globally, many upper gastrointestinal endoscopies are performed for inappropriate indications, and the overuse of healthcare negatively affects healthcare quality and places pressure on endoscopy services [34]. Therefore, the current systematic review and metaanalysis assessed the justifications as to whether p-EGD should be routinely undertaken for all BS patients. 


\section{Methods}

This systematic review and meta-analysis was conducted and reported according to the Preferred Reporting Items for Systematic Reviews and Meta-Analyses (PRISMA) Statement. The study was registered at the International prospective register of systematic reviews (PROSPERO CRD42020157596).

\section{Literature Searches}

A systematic review was carried out using PubMed, Cochrane Central Register of Controlled Trials (CENTRAL), WHO International Clinical Trials Registry Platform, Cochrane Library, MEDLINE, Scopus, clinicaltrials.gov, and Google scholar electronic databases. We used the keywords "bariatric surgery" "Esophagogastroduodenoscopy," "preoperative" [in Title/Abstract]. The medical subject headings $(\mathrm{MeSH})$ terms used were bariatric surgery (All Fields) AND "Esophagogastroduodenoscopy" (MeSH Terms); bariatric surgery (All Fields) AND "preoperative AND Esophagogastroduodenoscopy" (MeSH Terms); bariatric surgery (All Fields) AND "preoperative OR Esophagogastroduodenoscopy" (MeSH Terms). Additional searches were conducted using the reference lists of studies and review articles for a selection of relevant articles. The references of all included articles or relevant reviews were cross-checked.

\section{Inclusion/Exclusion Criteria}

The inclusion criteria were (1) original studies, (2) English language, (3) published from 01 January 2000 through 30th April 2019, (4) assessed "Esophagogastroduodenoscopy" and "bariatric surgery," and, (5) patients of any age, gender, and ethnicity. Articles other than original studies such as commentaries, letters to the editor, reviews, case reports, and studies that did not include outcomes or comparisons were also excluded. The consensus on the inclusion/exclusion criteria was premised on the fact that whether a given study provided information on the association between p-EGD and postoperative outcomes among bariatric surgery patients. Therefore, even studies with smaller sample sizes were also included in the initial evaluation. Three authors independently abstracted the data.

\section{Objectives}

To assess the significance of routine p-EGD screening in BS, the specific objectives were to:
- Conduct a systematic review of the literature in order to identify all relevant articles on the topic;

- Employ Sharaf et al.'s classification [6] of predetermined criteria to categorize the p-EGD findings of each article into the four groups (detailed below);

- Compute the yield of p-EGD findings of each article in terms of the four groups of Sharaf et al.'s classification [6]; and,

- Use the findings emerging from the meta-analysis to make informed judgments of the justification as to whether $\mathrm{p}$ EGD should be routinely undertaken for all BS patients or otherwise.

\section{Categorization of P-EGD Findings}

In order to gauge the value of routine p-EGD screening in BS, we employed Sharaf et al.'s classification [6] of predetermined criteria to categorize $\mathrm{p}$-EGD findings into four groups:

Group 0: no abnormal p-EGD findings, i.e., normal. Group 1: abnormal p-EGD findings that do not necessitate changing the surgical approach or postponing surgery (e.g., mild esophagitis, gastritis and/or duodenitis, esophageal web).

Group 2: abnormal p-EGD findings that change the surgical approach or postpone surgery (e.g., mucosal/ submucosal mass lesions, ulcers, severe erosive esophagitis, gastritis, and/or duodenitis, Barrett's esophagus, Bezoar, hiatal hernia, peptic stricture, Zenker's or esophageal diverticula, arteriovenous malformations).

Group 3: p-EGD findings that signify absolute contraindications to surgery (e.g., upper gastrointestinal cancers and varices).

\section{Data Extraction}

The titles of the research articles obtained from the initial database searches were screened and relevant papers were selected. Then the abstracts and full texts were reviewed according to the inclusion criteria for final selection. Three authors independently reviewed the studies based on the exclusion and inclusion criteria. Initially, titles of the studies identified from the search were assessed for inclusion. Titles approved by the authors were moved to abstract screening. If three authors rejected a study at this stage, it was excluded 
from the review. In the third stage, full text articles were screened for eligibility. Only those studies approved by the three authors were included in the review. Agreement between the authors on the quality of the articles ranged between 90 and $100 \%$. All disagreements were resolved by consensus among the authors. Data extracted from the selected articles included authors, the origin of studies, source population, study settings and duration, inclusion/exclusion criteria, data sources and measurement, sample size, and the yield of pEGD findings in terms of the four groups of Sharaf et al.'s classification [6].

\section{Methodological Quality}

The methodological quality of the selected studies was assessed based on five STROBE criteria from the checklist, namely, study design, setting, participants, data sources/measurement, and study size. The STROBE checklist and the five criteria selected from the checklist were most relevant in the assessment of the methodological quality of observational studies in epidemiology (Table 1).

\section{Data Analysis and Synthesis}

Prevalences were calculated for categorical variables. The decision to employ either a fixed-effect or random effect model depended on the results of statistical tests for heterogeneity. Data heterogeneity was assessed using the Cochrane Q homogeneity test (significance set at $p<0.10$ ). If the studies were statistically homogeneous, a fixed-effect model was selected. A random effect model was used when studies were statistically heterogeneous. The Higgin's $I^{2}$ test is the ratio of true heterogeneity to the total variation in observed effects. A rough guide to interpretation of $I^{2}$ test is $0-25 \%$ : might not be important; 25-50\%: may represent moderate heterogeneity; 50-75\%: may represent substantial heterogeneity; and > 75\%: considerable heterogeneity. Publication bias was visually estimated by assessing funnel plots. Pooled estimates were calculated using the R 3.5.1 software.

\section{Results}

The search generated a total of 1256 articles; 1209 articles were either non-relevant to the topic, duplicates, or review articles which were excluded. The relevant titles and/or abstracts and full text of the remaining 47 articles underwent detailed evaluation, after which 22 articles were further eliminated as these were mainly based on protocol development and narrative reviews. Finally, 25 original studies met all the review criteria and were considered for the final meta-analysis
(Fig. 1 and Table 1) $[2,6,8,9,11,14,18,21-25,28,30$, 35-45].

Median study duration was 4 years with an inter quartile range of 2-7 years. Overall average age was 40.7 years, and overall average percentage of males $(25 \%)$ was lower than females $(75 \%)$. All studies were non-randomized controlled trials, comprising 15 prospective and 10 retrospective studies. These studies had low or unclear risk of bias, unlikely to seriously alter the results. In addition, these studies had no serious risk of bias that can downgrade the quality. There was no inconsistency: the study populations were BS patients, and outcome assessment was consistent, namely the yield of p-EGD findings in terms of the four groups of Sharaf et al.'s classification [6].

\section{Outcome Measures}

The total number of patients pooled was 10,685 . Figure 2 depicts the meta-analysis of the 4 groups (groups $0-3$ ) of patients based on their p-EGD findings. The largest group was Group 0 (no abnormal p-EGD findings, 56\%, 95\% CI: 45-67\%) followed by Group 1 (abnormal p-EGD findings that do not necessitate changing the surgical approach or postponing surgery, $26 \%, 95 \%$ CI: $18-35 \%)$. These were followed by Group 2 (abnormal p-EGD findings that change the surgical approach or postpone surgery, $16 \%, 95 \%$ CI: $11-$ $21 \%$ ) and Group 3 (p-EGD findings that signify absolute contraindications to surgery, $0.4 \%, 95 \%$ CI: $0-1 \%$ ). H. pylori infection was positive among about one-fourth of patients, and hiatal hernia was present in a mean of $17 \%$ of patients.

\section{Heterogeneity Among Included Studies}

The results for the test of heterogeneity for the meta-analysis among bariatric surgery patients are displayed in the bottom line to the left of each Forest plot. For Group 0 (no abnormal p-EGD findings), $Q\left[\chi^{2}\right]=1285.41, P=0.001, I^{2}=99 \%$, $\operatorname{tau}^{2}=0.0159$ (Fig. 2a); for Group 1 (abnormal p-EGD findings that do not necessitate changing the surgical approach or postponing surgery), $Q\left[\chi^{2}\right]=165.03, P=0.001, I^{2}=99 \%$, $\operatorname{tau}^{2}=0.140$ (Fig. 2b); for Group 2 (abnormal p-EGD findings that change the surgical approach or postpone surgery), $Q$ $\left[\chi^{2}\right]=557.02, P=0.001, I^{2}=97 \%$ tau $^{2}=0.077$ (Fig. 2c); for Group 3 (p-EGD findings that signify absolute contraindications to surgery) $Q[\chi 2]=557.02, P=0.001$, $I^{2}=72 \%, \operatorname{tau}^{2}=0.007$ (Fig. 2d); for $H$ pylori infection $Q$ $\left[\chi^{2}\right]=1207.84, P=0.001, I^{2}=98 \%$, tau $^{2}=0.007$ (Fig. 2e); and, for hiatal hernia $Q\left[\chi^{2}\right]=556.10, P=0.001, I^{2}=96 \%$, $\operatorname{tau}^{2}=0.196$ (Fig. 2f). However, as $I^{2}$ was $>25 \%$, a random effect model was considered. Tau ${ }^{2}$ reflect the amount of true heterogeneity among the studies. 


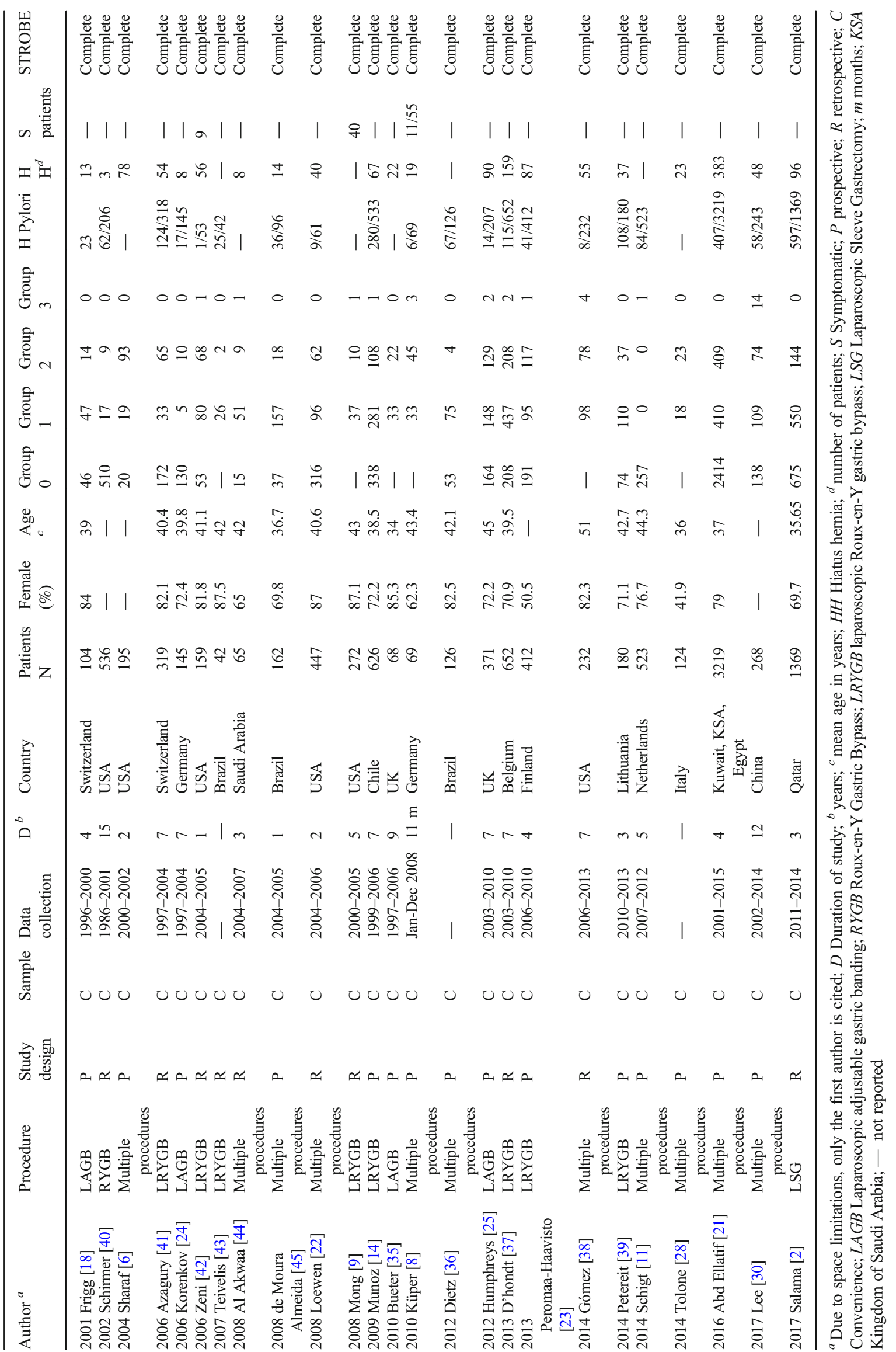


Fig. 1 Flow diagram of study selection process for systematic review

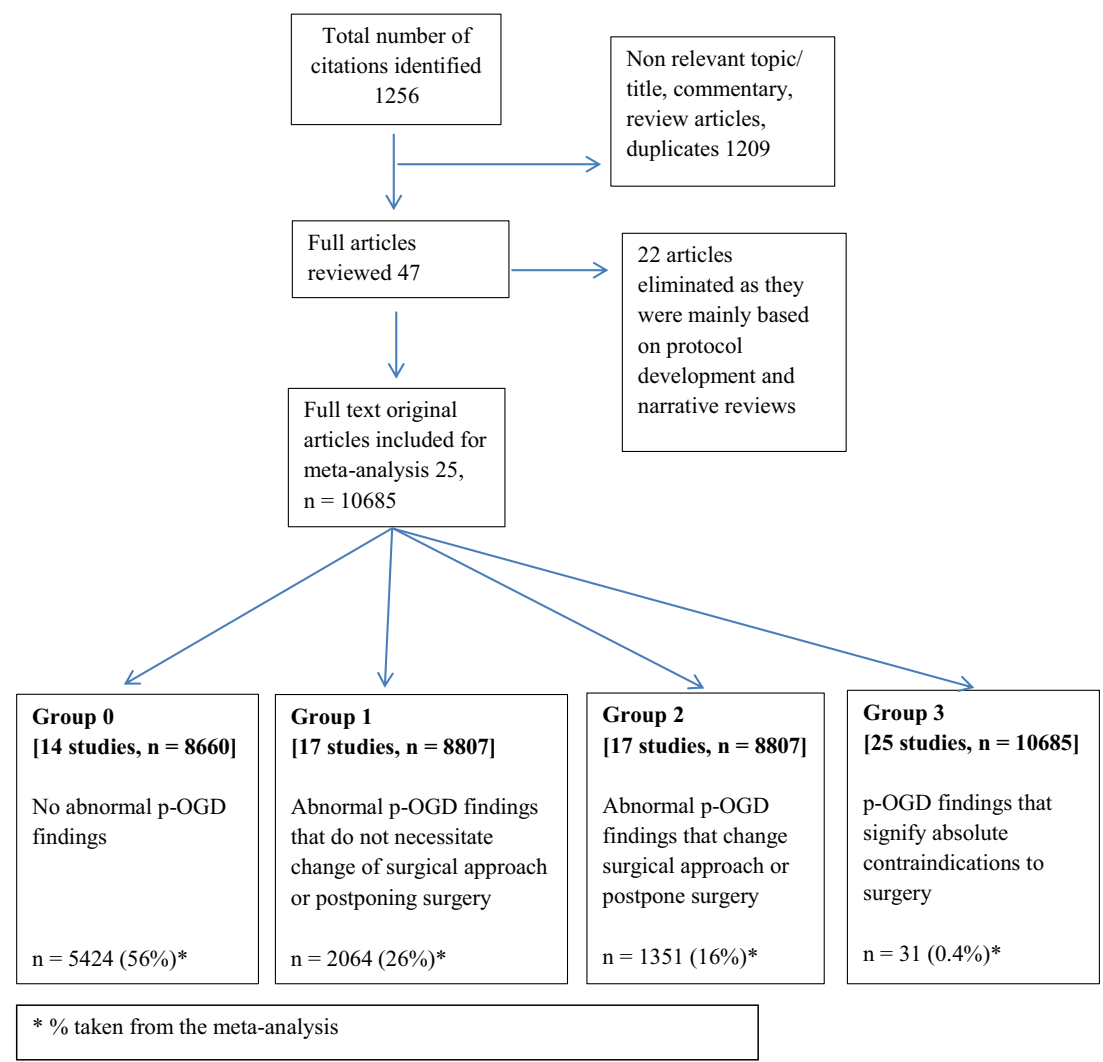

\section{Publication Bias and Funnel Plots}

For all of the above analyses, sensitivity analysis yielded consistent results. Based on a visual inspection of the funnel plots, there was evidence of publication bias for the included studies (Fig. 3). The funnel plots exhibited presence of studies with large standard error and they were not symmetrical.

\section{Limitation}

The studies included in this meta-analysis did not report the frequency of multiple abdominal conditions. Rather, the studies reported the frequency of each abdominal condition separately. Hence, there might be a probability of multiple abdominal conditions for a single patient which would influence the overall estimation in Groups 1 and 2.

\section{Discussion}

The current systematic review and meta-analysis is the first to assess the yield of p-EGD findings in terms of four groups [6], in order to gauge justifications as to whether p-EGD should be routine for all BS patients. Routine p-EGD can diagnose rare gastric pathologies
[19]. The current review showed that $82 \%$ of patients had either no abnormal p-EGD findings (Group 0) or abnormal $\mathrm{p}$-EGD findings that do not necessitate changing the surgical approach or postponing surgery (Group 1). Another $16 \%$ of patients required changing the surgical approach or postponing surgery based on the p-EGD findings (Group 2). Only $0.4 \%$ of patients had p-EGD findings that signified absolute contraindication to surgery (Group 3).

Generally, EGD carries risks to patients, as well as legal risks [46]. Hence, in addition to the p-EGD 'yield' in discovering/excluding pathologies, the appropriate gauging of whether routine p-EGD is justified for all BS patients needs to consider several parameters. These include the following: adverse effects of routine p-EGD; missing or over-diagnoses of lesions (false negatives, false positives); skill level of the esophagogastroduodenoscopy personnel; availability and cost of alternative (non-invasive) diagnostic methods to discover upper gastrointestinal pathology; and the costs of routine p-EGD. A related point is the changes that could occur to any missed pathology across time: i.e., initially before and then subsequent to BS (histological patterns of cellular alterations after gastric surgeries).

Adverse effects of esophagogastroduodenoscopy include infections, bleedings or perforations [47, 48], acute pancreatitis (direct trauma/gas insufflation) [49]; cardiopulmonary events 
a

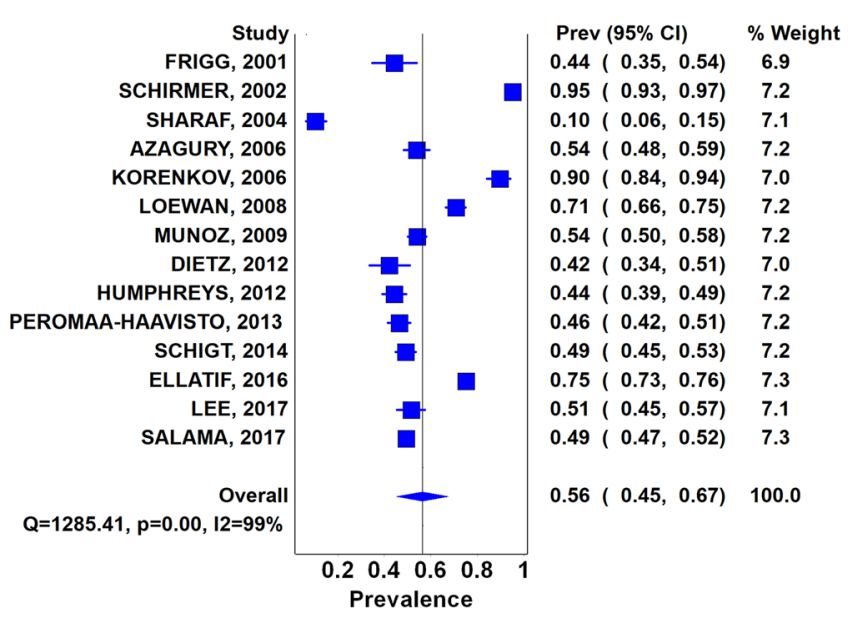

b
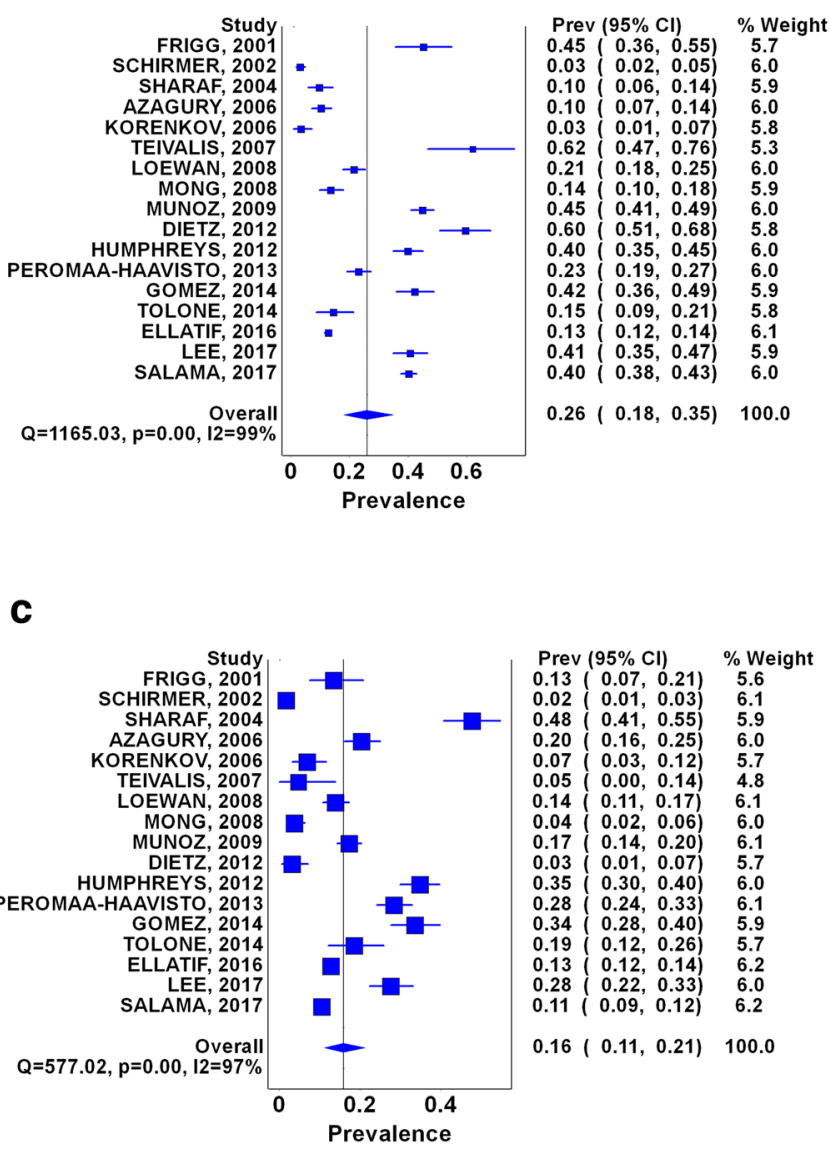

Fig. 2 Forest plots of a no abnormal p-EGD findings (Group 0); b abnormal p-EGD findings that do not necessitate changing the surgical approach (Group 1); $\mathbf{c}$ abnormal p-EGD findings that change the surgical

[48]; methemoglobinemia (genetic predispositions/use of topical anesthetics) [50]; hypoxic respiratory failure/critical events requiring bronchoscopic intratracheal oxygen insufflation $[8$, 51]; orbital hematoma [52]; and Takotsubo cardiomyopathy
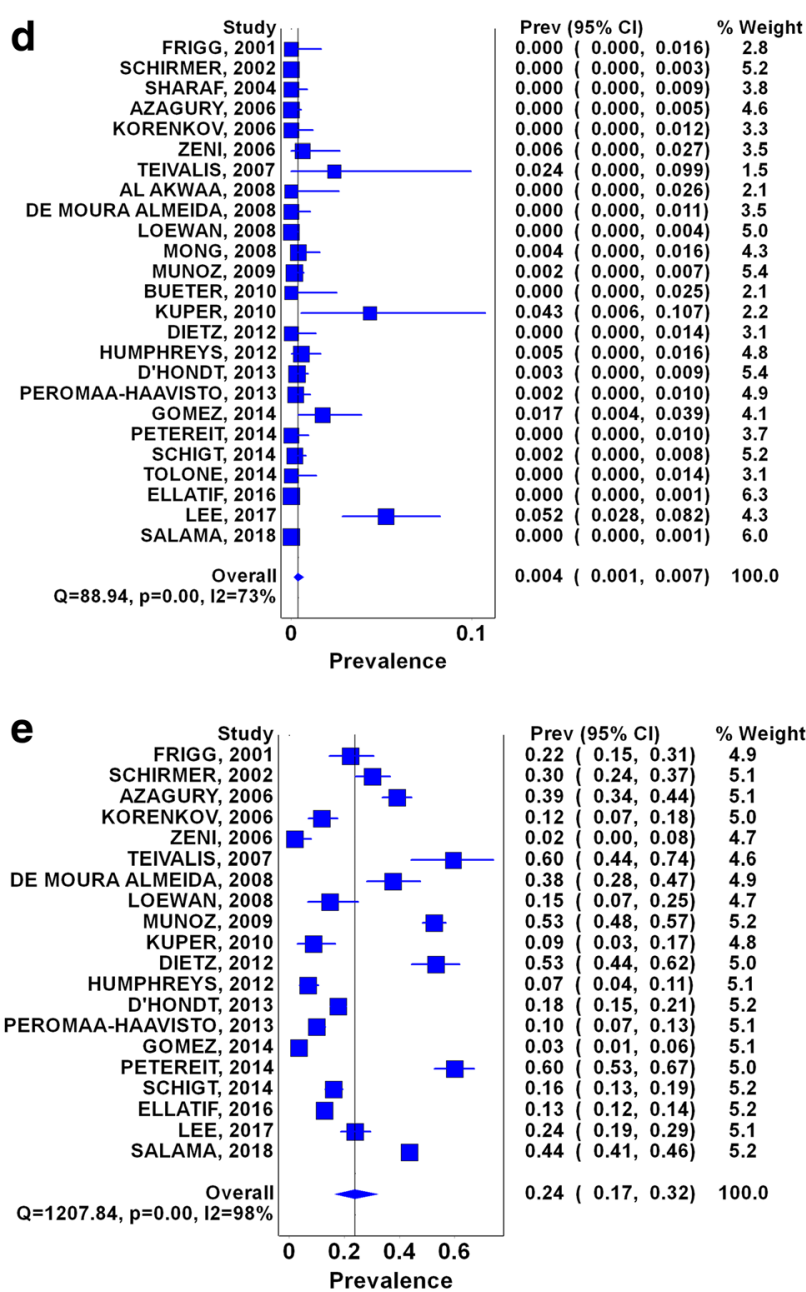

Prev $(95 \%$ CI) $0.22(0.15,0.31) \quad 4.9$ $\begin{array}{ll}0.30 & (0.24,0.37) \\ 0.39 & 5.1\end{array}$ $0.39(0.34,0.44) \quad 5.1$ $0.12(0.07,0.18) \quad 5.0$ $0.60(0.44,0.74) \quad 4.6$ $\begin{array}{lll}0.60 & (0.44,0.74) & 4.6 \\ 0.38 & (0.28,0.47) & 4.9\end{array}$ $\begin{array}{lll}0.15 & (0.28,0.47) & 4.9 \\ 0.07 & 0.25) & 4.7\end{array}$ $\begin{array}{lll}0.53(0.48,0.57) \quad 5.2 \\ 0.53(0.44,0.62) & 5.0\end{array}$ $0.53(0.44,0.62) \quad 5.8$ $0.07(0.04,0.11) \quad 5.1$ $0.18(0.15,0.21) \quad 5.1$ $0.10(0.07,0.13) \quad 5.1$ $0.03(0.01,0.06) \quad 5.1$ $0.60(0.53,0.67) \quad 5.0$ $0.16(0.13,0.19) \quad 5.2$ $0.13(0.12,0.14) \quad 5.2$ $0.24(0.19,0.29) \quad 5.1$ $0.24(0.17,0.32) \quad 100.0$
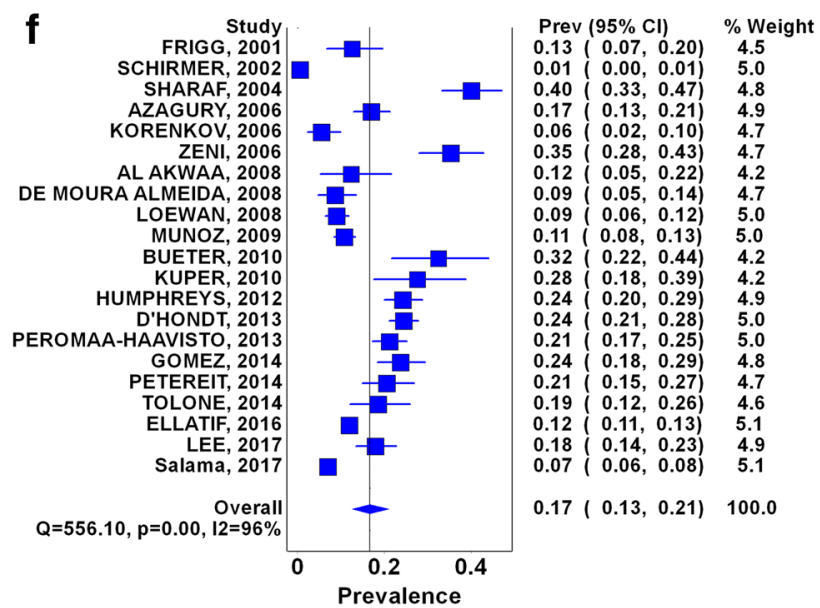

approach or postpone surgery (Group 2); d p-EGD findings that signify absolute contraindications to surgery (Group 3); e H. pylori infection; f Hiatal hernia

with complete heart block [53]. Other effects include preendoscopy anxiety (unsedated esophagogastroduodenoscopy) [54], effects related to comorbidities of e.g., morbidly obese diabetic patients where the overnight fasting challenges the 

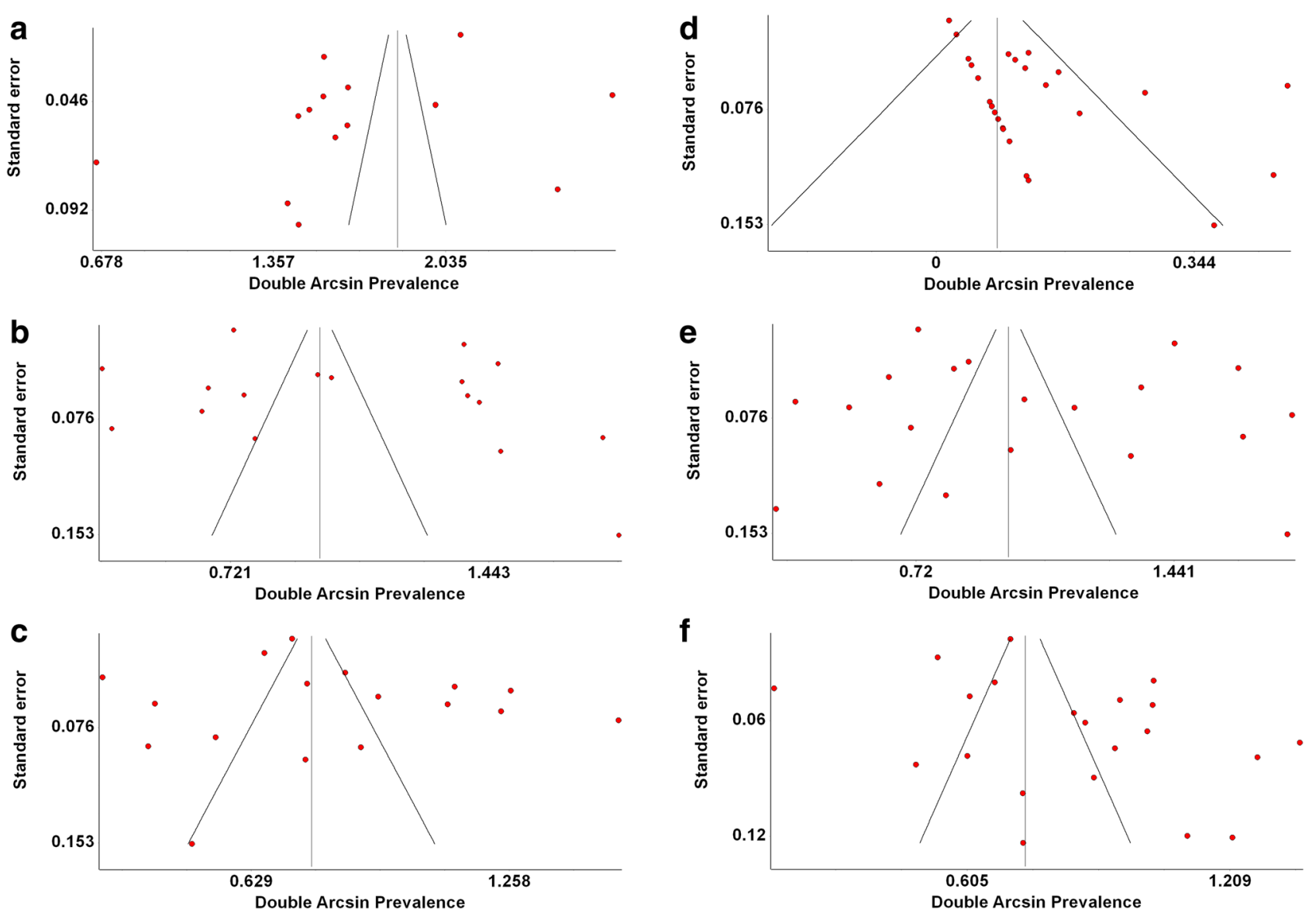

Fig. 3 Funnel plots of a no abnormal p-EGD findings (Group 0); b abnormal p-EGD findings that do not necessitate changing the surgical approach (Group 1); c abnormal p-EGD findings that change the surgical

metabolic status, and sleep apnea (needs surveillance during sedation) [8]. Despite these, some authors suggest that the infrequent adverse events should not limit routine p-EGD [55].

As for missing important lesions (false negatives), the quality of the esophagogastroduodenoscopy varies [56]. In Spain, 17 out of 187 gastric cancer patients had prior esophagogastroduodenoscopy (9.1\%), and 12 of those 17 missed gastric cancer had prior esophagogastroduodenoscopy with abnormal findings [57]. P-EGD is also frequently inaccurate at diagnosing hiatal hernia (particularly large hernias), where 23 patients undergoing sleeve gastrectomy had paraesophageal hernia intraoperatively; many of these patients were asymptomatic, and p-EGD revealed large hiatal hernia in only 4 patients [58-60]. Conversely, hiatal hernia repair was performed in $56(5 \%)$ of patients positive for intraoperative findings despite a negative p-EGD for hiatal hernia [55]. A related point here pertains to the probability of changes of a given missed lesion, i.e., the changes of pathology across time and the histological cellular alterations after gastric surgeries [61]. Pre-surgery biopsies of 798 LSG patients showed nonsignificant findings in $86.2 \%$; among them, $99.7 \%$ maintained a pattern without relevance for its follow-up; and some

approach or postpone surgery (Group 2); d p-EGD findings that signify absolute contraindications to surgery (Group 3); e H. pylori infection; f Hiatal hernia

patients who had intestinal metaplasia reversed its histopathology (maybe following H. pylori treatment) [62]. Others found that the pre-operative inflammatory alterations were reduced post-operatively, where the chronic gastritis with inflammatory activity associated with $H$. pylori was reduced by $16.7 \%$, and foveolar hyperplasia was reduced by $25 \%$ [61]. Further research can evaluate whether such improvements are due to treatment of $H$. pylori [61].

In terms of false positives, EGD over-diagnosed small hiatal hernias, most did not require repair, and $60 \%$ of EGD positive hiatal hernias were found to be negative intraoperatively [55]. Both the presence of symptoms and EGD findings may not always correlate with intraoperative findings [55]. In the current meta-analysis, p-EGD findings suggested hiatal hernia in a mean of $17 \%$ of patients (95\% CI: $13-21 \%$ ). However, the data provided by the studies does not enable one to speculate how many hiatal hernias/other lesions were missed or over-diagnosed during these EGDs.

In connection with the skill level, p-EGD has some subjectivity; hence, the endoscopist's expertise could lead to over/under diagnoses $[55,63]$. The endoscopist is vital in missed gastric cancer [57], and training/learning interventions 
could enhance the quality of endoscopy [63]. About $51.8 \%$ of the incomplete endoscopy reports did not have justification for its incompleteness [64]. Patients with no symptoms or no esophagogastroduodenoscopy evidence of hiatal hernias had hernia repairs $(4 \%-6 \%)$, suggesting that small hiatal hernias are operator-dependent diagnoses [55]. The studies included in the current meta-analysis did not examine such skills, and we are unable to conclude how this might have affected the $\mathrm{p}$ EGD yield we computed.

In terms of alternative diagnostic methods for gastric cancer pathologies, there are novel noninvasive screening techniques for e.g., Barrett's esophagus [65] and H. pylori [66-68]. However, some authors might view that some novel techniques might be inferior to established gold standards, not all institutions might have advanced alternative diagnostic technologies, and esophagogastroduodenoscopy allows both the direct visualization and tissue biopsy [55].

Endoscopy is costly [1]. In the USA, the average hospital cost of an esophagogastroduodenoscopy with and without biopsy was \$3732 and \$3038 [69]. Endoscopy necessitates time, money, and personnel resources including experienced investigators, anesthesiological support, and special surveillance [8].

The current meta-analysis found that Group 2 patients (abnormal p-EGD findings that change the surgical approach or postpone surgery) amounted to $16 \%$. However, it is not clear what proportion of these patients were postponed solely for H. pylori medical treatment as opposed to a "true" more substantial esophagogastroduodenoscopy-informed change in the surgical approach. This is important, as some might argue that if $H$. pylori is diagnosed by a non-invasive method (no need for esophagogastroduodenoscopy), and if the surgery waiting list time at a given institution is $>2-4$ weeks (sufficient time for $H$. pylori treatment), then no postponement might have been required. One inquiry [2] examined the postponement, cancelation, or change of surgical approach based on the pEGD findings across several sleeve gastrectomy studies and found that a considerable number of Group 2 patients were postponed solely for the treatment of $H$. pylori. This research [2] reported that across three studies, 21.5\% [6], 12\% [10], and $27 \%$ [30] of Group 2 patients had their BS postponed for $H$. pylori treatment, or waiting for $H$. pylori test result to assess severity of inflammation after medical treatment. Such findings suggest, that for the present meta-analysis, it might be reasonable to speculate that the proportion of Group 2 patients postponed due to a "true" change in surgical approach could be much less that the current $16 \%$, further questioning the utility of routine $\mathrm{p}$-EGD.

This review searched most of the citation databases and reference lists of the included studies. We also accessed paid articles. Nevertheless, a limitation of the current meta-analysis is that it included only published studies and only the English literature. We could not find "gray" literature, and hence, potential publication bias cannot be excluded. There were no studies from some regions of the world. However, 25 studies were included in this meta-analysis and we had a sizeable sample of 10,685 patients.

\section{Conclusions}

The findings of this meta-analysis compel a revisit of current practice, and a re-evaluation of why p-EGD should be routine for all bariatric surgery patients. In 2016, about 634,897 bariatric operations were performed worldwide [70]. It might not be totally judicious to expose very large numbers of morbidly obese patients to a routine invasive uncomfortable procedure that has potential (although minimal) risk and insufficient evidence of effectiveness. Limitations include the lack of studies from some world regions and a small number of studies.

Authorship WEA was involved in the conceptualization and design of this study. AE, SB, and WEA searched databases, screened articles extracted data. SB performed the acquisition and analysis of data. $\mathrm{AE}, \mathrm{SB}$, and WEA interpreted the data. WEA AE, and SB drafted the manuscript. HAT, MA and AA critically revised the manuscript. All authors approved the final version of the manuscript. WEA is the guarantor of this study.

Funding Information Open Access funding for the publication of this article provided by Qatar National Library.

\section{Compliance with Ethical Standards}

Conflict of Interest The authors declare that they have no conflicts of interest.

Ethical Approval For this type of study, ethical approval and informed consent do not apply as it is a systematic review and meta-analysis.

Open Access This article is licensed under a Creative Commons Attribution 4.0 International License, which permits use, sharing, adaptation, distribution and reproduction in any medium or format, as long as you give appropriate credit to the original author(s) and the source, provide a link to the Creative Commons licence, and indicate if changes were made. The images or other third party material in this article are included in the article's Creative Commons licence, unless indicated otherwise in a credit line to the material. If material is not included in the article's Creative Commons licence and your intended use is not permitted by statutory regulation or exceeds the permitted use, you will need to obtain permission directly from the copyright holder. To view a copy of this licence, visit http://creativecommons.org/licenses/by/4.0/.

\section{References}

1. Lalor PF. Comment on: is esophagogastroduodenoscopy before Roux-en-Y gastric bypass or sleeve gastrectomy mandatory? Surg Obes Relat Dis. 2014;10(3):417-8.

2. Salama A, Saafan T, El Ansari W, et al. Is routine preoperative esophagogastroduodenoscopy screening necessary prior to laparoscopic sleeve gastrectomy? Review of 1555 Cases and Comparison 
with Current Literature. Obes Surg. 2018;28(1):52-60. Erratum in: Obes Surg. 2017 Nov;27(11):3068

3. Sauerland S, Angrisani L, Belachew M, et al. Obesity surgery: evidence-based guidelines of the European Association for Endoscopic Surgery (EAES). Surg Endosc. 2005;19:200-21.

4. SAGES Guidelines Committee. SAGES guideline for clinical application of laparoscopic bariatric surgery. Surg Obes Relat Dis. 2009;5(3):387-405.

5. SICOB Linee Guida di Chirurgia dell' Obesita. Società Italiana di Chirurgia dell'Obesità e della Malattie Metaboliche. 2016 edition Available from: https://www.sicob.org/00_materiali/linee_guida 2016.pdf (accessed 17 July 2019)

6. Sharaf RN, Weinshel EH, Bini EJ, et al. Endoscopy plays an important preoperative role in bariatric surgery. Obes Surg. 2004;14: 1367-72.

7. Verset D, Houben JJ, Gay F, et al. The place of upper gastrointestinal tract endoscopy before and after vertical banded gastroplasty for morbid obesity. Dig Dis Sci. 1997;42:2333-7.

8. Küper MA, Kratt T, Kramer KM, et al. Effort, safety, and findings of routine preoperative endoscopic evaluation of morbidly obese patients undergoing bariatric surgery. Surg Endosc. 2010;24(8): 1996-2001.

9. Mong C, Van Dam J, Morton J, et al. Preoperative endoscopic screening for laparoscopic Roux-en-Y gastric bypass has a low yield for anatomic findings. Obes Surg. 2008;18(9):1067-73.

10. Praveenraj P, Gomes RM, Kumar S, et al. Diagnostic yield and clinical implications of preoperative upper gastrointestinal endoscopy in morbidly obese patients undergoing bariatric surgery. J Laparoendosc Adv Surg Tech A. 2015;25(6):465-9.

11. Schigt A, Coblijn U, Lagarde $\mathrm{S}$, et al. Is esophagogastroduodenoscopy before Roux-en-Y gastric bypass or sleeve gastrectomy mandatory? Surg Obes Relat Dis. 2014;10(3):411-7. quiz 565-6

12. Lord RV, Edwards PD, Coleman MJ. Gastric cancer in the bypassed segment after operation for morbid obesity. Aust N Z J Surg. 1997;67:580-2.

13. Khitin L, Roses RE, Birkett DH. Cancer in the gastric remnant after gastric bypass. Curr Surg. 2003;60:521-3.

14. Munoz R, Ibanez L, Salinas J, et al. Importance of routine preoperative upper GI endoscopy: why all patients should be evaluated? Obes Surg. 2009;19:427-31.

15. Seva-Pereira G, Trombeta VL. Early gastric cancer found at preoperative assessment for bariatric surgery. Obes Surg. 2006;16:110911.

16. Boru C, Silecchia G, Pecchia A, et al. Prevalence of cancer in Italian obese patients referred for bariatric surgery. Obes Surg. 2005;15: 1171-6.

17. Cowan GSM, Hiler ML. Upper gastrointestinal endoscopy in bariatric surgery. In: Deitel M, editor. Update: Surgery for the Morbidly Obese Patient. Toronto: FD-Communications Inc; 2000. p. 387-416.

18. Frigg A, Peterli R, Zynamon A, et al. Radiologic and endoscopic evaluation for laparoscopic adjustable gastric banding: preoperative and follow-up. Obes Surg. 2001;11:594-9.

19. Dogan U, Suren D, Oruc MT, et al. Spectrum of gastric histopathologies in morbidly obese Turkish patients undergoing laparoscopic sleeve gastrectomy. Eur Rev Med Pharmacol Sci. 2017;21(23):5430-6.

20. Anderson MA, Gan SI, Fanelli RD, et al. Role of endoscopy in the bariatric surgery patient. Gastrointest Endosc. 2008;68:1-10.

21. Abd Ellatif ME, Alfalah H, Asker WA, et al. Place of upper endoscopy before and after bariatric surgery: a multicenter experience with 3219 patients. World J Gastrointest Endosc. 2016;8(10): 409-17.

22. Loewen M, Giovanni J, Barba C. Screening endoscopy before bariatric surgery: a series of 448 patients. Surg Obes Relat Dis. 2008;4(6):709-12.
23. Peromaa-Haavisto $\mathrm{P}$, Victorzon M. Is routine preoperative upper GI endoscopy needed prior to gastric bypass? Obes Surg. 2013;23: 736-9.

24. Korenkov M, Sauerland S, Shah S, et al. Is routine preoperative upper endoscopy in gastric banding patients really necessary? Obes Surg. 2006;16(1):45-7.

25. Humphreys LM, Meredith H, Morgan J, et al. Detection of asymptomatic adenocarcinoma at endoscopy prior to gastric banding justifies routine endoscopy. Obes Surg. 2012;22(4):594-6.

26. Masci E, Viaggi P, Mangiavillano B, et al. No increase in prevalence of Barrett's oesophagus in a surgical series of obese patients referred for laparoscopic gastric banding. Dig Liver Dis. 2011;43(8):613-5.

27. Corley DA, Kubo A. Body mass index and gastroesophageal reflux disease: a systematic review and meta-analysis. Am J Gastroenterol. 2006;108:2619e2628.

28. Tolone S, Limongelli P, del Genio G, et al. Gastroesophageal reflux disease and obesity: do we need to perform reflux testing in all candidates to bariatric surgery? Int J Surg. 2014;12(Suppl 1): S173-7.

29. Howlader N, Noone AM, Krapcho M, Garshell J, Miller D, Altekruse SF, Kosary CL, Yu M, Ruhl J, Tatalovich Z, Mariotto A, Lewis DR, Chen HS, Feuer EJ, Cronin KA (eds). SEER Cancer Statistics Review, 1975-2012, National Cancer Institute. Bethesda, MD; [updated 2015 April; cited 2019 Dec 12] Available from http://seer.cancer.gov/csr/1975_2012/.

30. Lee J, Wong SK, Liu SY, et al. Is preoperative upper gastrointestinal endoscopy in obese patients undergoing bariatric surgery mandatory? An Asian Perspective. Obes Surg. 2017;27(1):44-50.

31. Yardimci E, Bozkurt S, Baskoy L, et al. Rare entities of histopathological findings in 755 sleeve gastrectomy cases: a synopsis of preoperative endoscopy findings and histological evaluation of the specimen. Obes Surg. 2018;28(5):1289-95.

32. Ge L, Moon RC, Nguyen H, et al. Pathologic findings of the removed stomach during sleeve gastrectomy. Surg Endosc. 2019;33: 4003-7. https://doi.org/10.1007/s00464-019-06689-y. [Epub ahead of print].

33. Coblijn UK, Kuiken SD, van Wagensveld BA. Comment on: is esophagogastroduodenoscopy before Roux-en-Y gastric bypass or sleeve gastrectomy mandatory? Surg Obes Relat Dis. 2015;11(5): $1192-3$

34. de Jong JJ, Lantinga MA, Drenth JP. Prevention of overuse: a view on upper gastrointestinal endoscopy. World J Gastroenterol. 2019;25(2):178-89.

35. Bueter M, Thalheimer A, Le Roux CW, et al. Upper gastrointestinal investigations before gastric banding. Surg Endosc. 2010;24(5): 1025-30.

36. Dietz J, Ulbrich-Kulcynski JM, Souto KEP, et al. Prevalence of upper digestive endoscopy and gastric histopathology findings in morbidly obese patients. Arq Gastroenterol. 2012;49(1):52-5.

37. D'Hondt M, Steverlynck M, Pottel H, et al. Value of preoperative esophagogastroduodenoscopy in morbidly obese patients undergoing laparoscopic Roux-en-Y gastric bypass. Acta Chir Belg. 2013;113(4):249-53.

38. Gómez V, Bhalla R, Heckman MG, et al. Routine screening endoscopy before bariatric surgery: is it necessary? Bariatr Surg Pract Patient Care. 2014;9(4):143-9.

39. Petereit R, Jonaitis L, Kupc `inskas L, et al. Gastrointestinal symptoms and eating behavior among morbidly obese patients undergoing Roux-en-Y gastric bypass. Medicina (Kaunas). 2014;50(2): 118-23.

40. Schirmer B, Erenoglu C, Miller A. Flexible endoscopy in the management of patients undergoing Roux-en-Y gastric bypass. Obes Surg. 2002;12(5):634-8. 
41. Azagury D, Dumonceau JM, Morel P, et al. Preoperative work-up in asymptomatic patients undergoing Roux-en-Y gastric bypass: is endoscopy mandatory? Obes Surg. 2006;16(10):1304-11.

42. Zeni TM, Frantzides CT, Mahr C, et al. Value of preoperative upper endoscopy in patients undergoing laparoscopic gastric bypass. Obes Surg. 2006;16(2):142-6.

43. Teivelis MP, Faintuch J, Ishida R, et al. Endoscopic and ultrasonographic evaluation before and after Roux-en-Y gastric bypass for morbid obesity. Arq Gastroenterol. 2007;44(1):8-13.

44. Al Akwaa AM, Alsalman A. Benefit of preoperative flexible endoscopy for patients undergoing weight-reduction surgery in Saudi Arabia. Saudi J Gastroenterol. 2008;14(1):12-4.

45. de Moura AA, Cotrim HP, Santos AS, et al. Preoperative upper gastrointestinal endoscopy in obese patients undergoing bariatric surgery: is it necessary? Surg Obes Relat Dis. 2008;4(2):144-9.

46. Adler DG. Consent, common adverse events, and post-adverse event actions in endoscopy. Gastrointest Endosc Clin N Am. 2015;25(1):1-8.

47. Jałocha L, Wojtuń S, Gil J. Incidence and prevention methods of complications of gastrointestinal endoscopy procedures. Pol Merkur Lekarski. 2007;22(131):495-8.

48. American Society for Gastrointestinal Endoscopy (ASGE) Standards of Practice Committee, Ben-Menachem T, Decker GA, et al. Adverse events of upper GI endoscopy. Gastrointest Endosc. 2012;76(4):1063-72.

49. Nwafo NA. Acute pancreatitis following oesophagogastroduodenoscopy. BMJ Case Rep. 2017;2017:bcr-2017-222272.

50. Khan K, White-Gittens I, Saeed S, et al. Benzocaine-induced methemoglobinemia in a postoperative bariatric patient following esophagogastroduodenoscopy. Case Rep Crit Care. 2019;2019: 1571423. eCollection 2019

51. Wiens EJ, Mylnikov A. Hypoxic respiratory failure complicating esophagogastroduodenoscopy. Am J Gastroenterol. 2019;114(5): 706.

52. Lotlikar M, Pandey V, Chauhan S, et al. Orbital hematoma: a new complication of esophagogastroduodenoscopy. Am J Gastroenterol. 2019;114(1):181-2.

53. Zhang AN, Sacchi $T$, Altschul R, et al. A case of esophagogastroduodenoscopy induced Takotsubo cardiomyopathy with complete heart block. Clin J Gastroenterol. 2019;12: 296-300. https://doi.org/10.1007/s12328-019-00967-x. [Epub ahead of print].

54. Yang M, Lu LL, Zhao M, et al. Associations of anxiety with discomfort and tolerance in Chinese patients undergoing esophagogastroduodenoscopy. PLoS One. 2019;14(2):e0212180.

55. Mohammed R, Fei P, Phu J, et al. Efficiency of preoperative esophagogastroduodenoscopy in identifying operable hiatal hernia for bariatric surgery patients. Surg Obes Relat Dis. 2017;13(2): 287-90.

56. Wang LW, Lin H, Xin L, et al. Establishing a model to measure and predict the quality of gastrointestinal endoscopy. World $\mathrm{J}$ Gastroenterol. 2019;25(8):1024-30.
57. Delgado Guillena PG, Morales Alvarado VJ, Jimeno Ramiro M, et al. Gastric cancer missed at esophagogastroduodenoscopy in a well-defined Spanish population. Dig Liver Dis. 2019; 51(8): 1123-9.

58. Pham DV, Protyniak B, Binenbaum SJ, et al. Simultaneous laparoscopic paraesophageal hernia repair and sleeve gastrectomy in the morbidly obese. Surg Obes Relat Dis. 2014;10(2):257-61.

59. Daes J, Jimenez ME, Said N, et al. Laparoscopic sleeve gastrectomy: symptoms of gastroesophageal reflux can be reduced by changes in surgical technique. Obes Surg. 2012;22:1874-9.

60. Soricelli E, Casella G, Rizzello M, et al. Initial experience with laparoscopic crural closure in the management of hiatal hernia in obese patients undergoing sleeve gastrectomy. Obes Surg. 2010;20: 1149-53.

61. Onzi TR, d'Acampora AJ, de Araújo FM, et al. Gastric histopathology in laparoscopic sleeve gastrectomy: pre- and post-operative comparison. Obes Surg. 2014;24(3):371-6.

62. Canil AM, Iossa A, Termine P, et al. Histopathology findings in patients undergoing laparoscopic sleeve Gastrectomy. Obes Surg. 2018;28(6):1760-5.

63. Wu L, Zhang J, Zhou W, et al. Randomised controlled trial of WISENSE, a real-time quality improving system for monitoring blind spots during esophagogastroduodenoscopy. Gut. 2019; 68(12): 2161-9.

64. Lisboa-Gonçalves P, Libânio D, Marques-Antunes J, et al. Quality of reporting in upper gastrointestinal endoscopy: effect of a simple audit intervention. GE Port J Gastroenterol. 2018;26(1):24-32.

65. Sanghi V, Thota PN. Barrett's esophagus: novel strategies for screening and surveillance. Ther Adv Chronic Dis. 2019;10: 2040622319837851.

66. Guarner J, Kalach N, Elitsur Y, et al. Helicobacter pylori diagnostic tests in children: review of the literature from 1999 to 2009. Eur J Pediatr. 2010;169:15-25.

67. Erzin Y, Altun S, Dobrucali A, et al. Evaluation of two enzyme immunoassays for detecting Helicobacter pylori in stool specimens of dyspeptic patients after eradication therapy. J Med Microbiol. 2005;54:863-6.

68. Erzin Y, Altun S, Dobrucali A, et al. Comparison of two different stool antigen tests for the primary diagnosis of Helicobacter pylori infection in turkish patients with dyspepsia. Helicobacter. 2004;9: $657-62$.

69. Johnson JM, Carter TM, Schwartz RW, et al. P24: pre-operative upper endoscopy in patients undergoing laparoscopic Roux-en-Y gastric bypass is not mandatory. Surg Obes Relat Dis. 2007;3:307.

70. Angrisani L, Santonicola A, Iovino P, et al. IFSO worldwide survey 2016: primary, endoluminal, and revisional procedures. Obes Surg. 2018;28(12):3783-94. 2

Publisher's Note Springer Nature remains neutral with regard to jurisdictional claims in published maps and institutional affiliations. 